improvement from the $1994-1998$ era to the $1999-2005$ era $(31.0 \%$ in $1973-1993$ era, $33.0 \%$ in $1994-1998$ era, $34.1 \%$ in $1999-2005$ era). From the data, it appears that although AA may have similar responses to therapeutic agents for $\mathrm{MM}$, access to care may lead to outcome disparities.

The efficacy of high-dose chemotherapy followed by ASCT has also been evaluated in other nations not typically represented in the previously mentioned trials. In Japan, a study comparing 90 patients treated with ASCT versus 60 historical controls showed significant improvements in median survival (76 vs. 28 months, $p<0.0001) .^{108}$ A retrospective study of 86 patients with MM from China compared patients receiving ASCT to those patients that achieved a CR or PR without ASCT. ${ }^{109}$ In the patients that were treated with ASCT, 43\% achieved $\mathrm{CR}$ and a longer duration of response of 33 months was observed in comparison to 17 and 18 months in those patients in $\mathrm{CR}$ and $\mathrm{PR}$ without undergoing $\mathrm{ASCT}$, respectively. The experience in India has also shown substantial response and survival rates with ASCT in MM. A report of 143 patients treated from 1990 to 2009 showed a $83.3 \%$ response rate (CR $40.6 \%$, VGPR $25.9 \%$, PR $16.8 \%$ ) with a median event-free survival of 30 months and overall survival of 79 months. ${ }^{110}$ Similar survival rates were observed in a study of 26 MM patients from Mexico treated with intravenous melphalan followed by ASCT (median disease-free survival 38 months, median overall survival 86 months). ${ }^{11}$

Although one of the larger Phase III trials assessing the efficacy of a novel agent (bortezomib with melphalan and prednisone by San Miguel et al) included patients from South America and Asia, most of the previously mentioned studies showing efficacy of the novel agents were limited to MM patients from the United States and Europe. There have been several Phase II studies from Japan showing the clinical utility of thalidomide, bortezomib, and lenalidomide in the relapsed/refractory setting. One such study of 66 Japanese MM patients treated with low-dose thalidomide and dexamethasone showed overall response rates of $63.6 \%$ with progression-free and overall survivals of 6.2 and 25.4 months. ${ }^{112}$ In 25 patients with relapsed/refractory MM from Japan, lenalidomide plus dexamethasone was shown to be highly effective (PR or higher response seen in $100 \%$ of the patients) with two-thirds having grade 3 or 4 neutropenia. ${ }^{113}$ Bortezomib with dexamethasone has also shown efficacy in Japanese MM patients with relapsed/refractory disease based on a study of 88 patients showing an overall response rate of $66.9 \%$, median overall survival of 16.8 months, and progression-free survival of 6.8 months. ${ }^{114}$ Of note, the toxicity profile in Japanese patients treated with thalidomide and bortezomib have been different from European and United States studies. Thalidomide was shown to cause higher incidences of leukopenia $(41 \%)$ and grade 3 leukopenia $(11 \%)$ and lower rates of peripheral neuropathy and deep vein thrombosis in the Japanese MM patients. ${ }^{112}$ Pharmacokinetic studies suggest that there may be lower rates of clearance and volume of distribution, and higher area under the plasma concentration-time curve in Japanese patients with MM than Caucasian patients and may explain the differences in the toxicity profile. ${ }^{115}$ Life-threatening lung injury has been associated with bortezomib and possibly thalidomide therapy in Japanese patients with MM. ${ }^{116-118}$ In a cohort of 13 Japanese MM patients being treated with bortezomib at a single center, four developed severe pulmonary complications with two
ANSWER - (To Medical Image - page 19)

\section{Bone marrow necrosis}

Bone marrow biopsy shows fibrinoid necrosis, ghost cells with acute inflammation and macrophages. Diagnosis of myelodysplastic syndrome (refractory anemia with excess blasts) was made. Bone marrow necrosis (BMN) is a rare clinicopathologic entity with grave prognosis. It was first reported by Wade and Stevenson in $1941 .^{1}$ It is distinct from avascular necrosis of bone and bone marrow aplasia; and is characterized by necrosis of myeloid tissue and medullary stroma with loss of fat spaces.

Pain, fever and fatigue are common symptoms. Lactate dehydrogenase and alkaline phosphatase are found to be elevated in approximately $50 \%$ of the patients. In a study by Janssens $A M,{ }^{2}$ underlying malignancy was found in ninety-one percent of the patients. Hematologic malignancies are the most common causes of BMN. Other etiologies include solid tumors, infection, medications and sickle cell disease. Given its common association, extensive search for malignancy is indicated in patients presenting with unexplained bone marrow necrosis.

\section{REFERENCE}

1. Wade L, Stevenson L. Necrosis of bone marrow with fat embolism in sickle cell anemia. Am J Path. 1941;17:47-54.

2. Janssens AM, Offner FC, Van Hove WZ. Bone marrow necrosis. Cancer. 2000;88:1769-80.

deaths unrelated to progression of disease. A questionnaire-based report was soon published thereafter showing that seven of 46 patients (15.2\%) developed pulmonary complications either definitely or probably related to bortezomib. Of the seven patients, three died of respiratory failure.

Novel agents have also proven effective in Korea for treating MM patients either as single agents, combined with other chemotherapy agents, or with two novel agents with similar toxicity profiles to the western literature. A multicenter, retrospective study included 95 patients who received bortezomib only ( $n=38$ patients), bortezomib plus dexamethasone ( $n=34$ patients), and bortezomib plus thalidomide-containing regimen ( $\mathrm{n}=23$ patients) and showed an overall response rate of $65 \%$ (CR/nearCR $33 \%$, PR $32 \%) .{ }^{119}$ The use of novel agents for induction with two cycles of VAD followed by 2 cycles of bortezomib/thalidomide/dexamethasone followed by ASCT in newly diagnosed Korean MM patients showed a response rate of $97 \%$ with $\mathrm{CR} / \mathrm{nCR}$ of $27 \%$ and median time to progression of 20.3 months. ${ }^{120}$ For those deemed non-transplant eligible, the combination of bortezomib/thalidomide/dexamethasone induction followed by consolidation with melphalan/prednisolone/thalidomide was evaluated in 35 Korean patients with MM. ${ }^{121}$ Early responses were observed in $97 \%$ of patients with $30 \%$ having VGPR or higher responses. These response rates were observed in both high and standard-risk patients, based on cytogenetic abnormalities, with 